\title{
Parasite load and genotype are associated with clinical outcome of piroplasm-infected equines in Israel
}

\author{
Sharon Tirosh-Levy, Amir Steinman, Hadas Levy, Yotam Katz, Margarita Shtilman and Yuval Gottlieb* (D)
}

\begin{abstract}
Background: Equine piroplasmosis is a highly endemic protozoan disease of horses worldwide, caused by Theileria equi and Babesia caballi. While most horses in endemic areas are subclinically infected, the mechanisms leading to clinical outcome are vastly unknown. Moreover, since clinical signs of disease are not specific, and the prevalence in endemic areas is high, it is difficult to determine if equine piroplasmosis is the cause of disease. To identify possible mechanisms leading to the clinical outcome in an endemic area, we compared parasite loads and genotypes in clinically and subclinically infected horses.

Methods: Blood was collected from horses with clinical signs consistent with equine piroplasmosis, and from apparently healthy horses in Israel. Packed cell volume and total solids were measured. Quantitative and diagnostic polymerase chain reaction were used to identify, quantify and classify equine piroplasmosis infection. Phylogenetic analyses were used to determine the genotype of both parasites.
\end{abstract}

Results: For both parasites, clinical cases were associated with low mean packed cell volume and high mean parasite load $(P<0.001)$, enabling the determination of a cut-off value to distinguish between clinically and subclinically infected horses. Samples of Theileria equi from subclinical horses were classified into three different 185 rRNA genotypes, $\mathrm{D}(n=23), \mathrm{A}(n=12)$ and $\mathrm{C}(n=5)$, while samples from all clinical cases $(n=6)$ were classified as genotype $\mathrm{A}$. The sequences of T. equi equi merozoite antigens 1 (ema-1,n=9) and $2(e m a-2, n=11)$ genes were fairly conserved and did not differ between clinical and subclinical cases. Babesia caballi rhoptry associated protein-1 (rap-1) was classified into sub-genotypes A1 $(n=14)$ and A2 $(n=5)$ with no association to clinical outcome. Classification of the 185 rRNA gene (sub-genotypes B1 and B2) agreed with the rap-1 classification.

Conclusions: The results of this study suggest that quantification of parasite loads of infected horses may be used to distinguish between infections resulting in disease and subclinical cases. Although number of clinical cases is limited, we identified T. equi $18 \mathrm{~S}$ rRNA genotype A to be associated with clinical disease. This finding emphasizes the importance of in-depth genetic characterization of T. equi genotypes to identify possible markers for virulence.

Keywords: Theileria equi, Babesia caballi, Equine piroplasmosis, Phylogeny, Parasitemia, Clinical signs

*Correspondence: gottlieb.yuval@mail.huji.ac.il

Koret School of Veterinary Medicine, The Robert H. Smith Faculty

of Agriculture, Food and Environment, The Hebrew University

of Jerusalem, Rehovot, Israel

(C) The Author(s) 2020. This article is licensed under a Creative Commons Attribution 4.0 International License, which permits use, sharing, adaptation, distribution and reproduction in any medium or format, as long as you give appropriate credit to the original author(s) and the source, provide a link to the Creative Commons licence, and indicate if changes were made. The images or other third party material in this article are included in the article's Creative Commons licence, unless indicated otherwise in a credit line to the material. If material is not included in the article's Creative Commons licence and your intended use is not permitted by statutory regulation or exceeds the permitted use, you will need to obtain permission directly from the copyright holder. To view a copy of this licence, visit http://creativeco mmons.org/licenses/by/4.0/. The Creative Commons Public Domain Dedication waiver (http://creativecommons.org/publicdomain/ zero/1.0/) applies to the data made available in this article, unless otherwise stated in a credit line to the data. 


\section{Background}

Equine piroplasmosis (EP) is an important tick-borne disease of equids, caused by the hemoprotozoan apicomplexan parasites Theileria equi and Babesia caballi. Both parasites are endemic in most parts of the world: South America, Africa and most parts of Asia and Europe, including Israel [1-3]. Introduction of these parasites to non-endemic areas, in which suitable tick vectors are prevalent, may cause epizootic spread of disease [1, 4]. Theileria equi is usually more prevalent than B. caballi in endemic regions, has more severe clinical manifestation and may lead to lifelong infestation if untreated $[1,2]$. Economic consequences of infection include medical treatment and mortality of clinically infected animals, reduced performance of infected animals and restrictions on animal transport between endemic and non-endemic areas [1,2].

Clinical disease is usually characterized by acute hemolytic anemia, and ranges from subclinical, non-apparent parasite carriage to peracute, life-threatening disease. Clinical signs of acute disease include anemia, jaundice, inappetence, edema and pigmenturia. Most infected horses become asymptomatic carriers after resolution of clinical signs $[1,2]$. The factors contributing to the development of clinical disease are unclear. Host innate immunity plays a central role in the immune response, while adaptive immunity is also essential as high antibody titer correlates with parasite control $[1,5]$. Early exposure in endemic areas usually leads to protective immunity, while primary exposure of naive adults more often leads to clinical disease. However, clinical cases in adult horses are also reported in endemic areas $[1,2]$.

Apicomplexan parasites, including $T$. equi and $B$. caballi, show genetic diversity [6-9]. Five T. equi and two $B$. caballi $18 S$ rRNA genotypes have been described $[3,10]$, and none was associated with parasite virulence. In recent years, $18 S$ rRNA based classification has been questioned, as evidences for considerable genetic variation in other loci led to the description of several potential new species $[7,8]$. The novel species Theileria haneyi has $18 S$ rRNA sequence similar to genotype $\mathrm{C}$ but has a much smaller and considerably different genome compared to T. equi [8], and three variants of Theileria africa have $18 S$ rRNA sequences similar to genotype $\mathrm{D}$, but differs from T. equi in its $30 S$ rRNA gene sequence [7].

In order to detect genetic variation that is linked to parasite evasion of the host immune system, known immunogenic proteins have been characterized: in B. caballi, rhoptry associated protein-1 (rap-1) gene and protein [11-13] and in T. equi, equi merozoite antigen (ema) gene family, which includes nine genes [3, 14-16]. Two B. caballi rap-1 genotypes have been characterized with considerable differences in protein structure [11], jeopardizing the results of the United States Department of
Agriculture (USDA)-approved cELISA detection kit (based on the RAP-1 protein) on isolates from Africa and the Middle East [11-13]. The T. equi ema-1 and ema-2 genes and proteins are more conserved, and have been used for the development of serological assays [17, 18]. Some sequence variation has been detected for these genes, although little diversity is found among isolates within a geographical region $[3,14,19]$.

The aim of this study was to identify possible associations between parasite genotype and parasite density and the clinical outcome in T. equi and B. caballi infection in an endemic area, by comparing clinical with subclinical cases in Israel. The use of quantitative tools and additional parasite gene sequences were applied to overcome the limitations of classification based solely on the $18 S$ rRNA gene.

\section{Methods}

\section{Sample collection}

Veterinarians listed in the Israeli Association of Equine Practitioners were asked to send blood samples from any case suspected as EP. The clinical evaluation was left to the attending veterinarian's discretion. Most referred cases exhibited one or more of the following clinical signs: fever, anemia, icterus, inappetence and documented or suspected exposure to ticks. Blood samples $(n=25)$ were diagnosed molecularly as described bellow, and tested for packed cell volume (PCV), when available. Only horses with confirmed infection to either of the parasites by PCR and sequencing, were included in the study (six T. equi and six B. caballi).

Blood samples from apparently healthy horses were collected as a part of a surveillance study designed to represent the distribution of the horse population in Israel $(n=395)$. Blood from all horses was collected from the jugular vein into sterile vacuum tubes containing ethylenediamine tetraacetic acid (EDTA). Packed cell volume and total solids (TS) were measured using standard methodology prior to storage of whole blood at $-20{ }^{\circ} \mathrm{C}$ until processing. All blood samples were screened for the presence of both parasites using PCR. For B. caballi, all samples which were PCR-positive and successfully sequenced, were included in the analysis $(n=13)$. For $T$. equi, five samples were selected from each of surveillance farms showing more than five positive horses and were used for the molecular characterization. Forty samples with good quality sequences were ultimately used in the analysis. The characteristics of all horses included in this study are listed in Additional file 1: Table S1.

\section{Identification and characterization of EP parasites using polymerase chain reaction (PCR)}

DNA was extracted as described in Tirosh Levy et al. [20], and initial screening for infestation with EP parasites was 
performed using diagnostic PCR directed to identify a 400-bp fragment of T. equi $18 \mathrm{~S}$ rRNA gene [21], and $B$. caballi rap-1 gene [11], as previously described [3, 12, 22] (for primer list, see Additional file 2: Table S2).

The molecular characterization of T. equi was based on amplification and sequencing of its $18 \mathrm{SRNA}$, ema-1 and ema-2 genes, while the molecular characterization of $B$. caballi was based on the amplification and sequencing of its $18 S$ rRNA and rap-1 genes. The full length (1600 bp) of T. equi and B. caballi $18 S$ rRNA gene was amplified using primers NBabesia1F and 18SRev-TB primers $[6,23,24]$ (Additional file 2: Table S2), as previously described [10]. Full-length sequences were amplified from all available clinical samples and $B$. caballi-positive samples and from 50 T. equi-positive subclinical samples, five horses per positive farm. A 750-bp fragment of $T$. equi ema-1 gene was amplified using primers EMA-1F/R [21] (Additional file 2: Table S2). A 800-bp fragment of $T$. equi ema-2 gene was amplified using primers EMA-2F/R (Additional file 2: Table S2). A fragment of approximately 1500-bp fragment of B. caballi rap-1 gene was amplified using the primers Bc9_RAPF/R, as previously described $[11,12]$ (Additional file 2: Table S2).

All positive PCR products were cleaned using Exonuclease I and Shrimp alkaline phosphatase (New England Biolabs Inc., Massachusetts, USA) and sent for sequencing (Macrogen Europe, Amsterdam, The Netherlands). Sequencing of the complete $18 S$ rRNA gene was performed using three different sets of primers, as previously described [3, 6] (Additional file 2: Table S2). Sequencing of ema-1 and ema-2 genes was performed using both the forward and reverse primers. Sequencing of rap-1 gene was performed using both the forward and reverse primers as well as the internal set of primers Bc9_RAP2F/R (Additional file 2: Table S2), as previously described [11, 12].

Consensus sequences of each sample and gene were constructed using the Chromas (version 2.6, Technelysium Pty Ltd., South Brisbane, Australia) and MEGA v.7.0.18 software [25] (http://www.megasoftware.net, last accessed November 2018), as previously described [20]. BLAST (http://www.ncbi.nlm.nih.gov/BLAST, last accessed November 2019) analysis confirmed that all constructed sequences were $99-100 \%$ identical to previously published sequences of the corresponding gene available on GenBank. The constructed sequences from all samples and genes were submitted to the GenBank database.

Phylogenetic trees were constructed using both Maximum Likelihood and Neighbor-Joining methods in MEGA7, as previously described [20]. The statistical model used for each analysis was selected by the lowest Bayesian Information Criterion (BIC) score, calculated in
MEGA7. All algorithms were constructed with bootstrap replicates from 1000 randomly selected samples to estimate reliability.

The divergence between T. equi ema-2 sequences from this study and from GenBank was estimated using distance matrix analysis, Kimura 2-parameter $+\mathrm{G}$ model in MEGA7.

\section{Quantification of EP parasitemia using quantitative real-time PCR reaction ( $q P C R$ )}

Quantification of parasitemia was assessed via $\mathrm{qPCR}$ using TaqMan ${ }^{\circledR}$ minor groove binder (MGB) (Thermo Fisher Scientific, Waltham, MA, USA) probes targeting T. equi ema-1 gene [14] and B. caballi $18 S$ rRNA gene [26] (Additional file 2: Table S2). A clean PCR product of each gene was used to prepare the standard curve, and gene copy number (gcn) was calculated from the molecular weight and gene length $[\mathrm{gcn}=(\mathrm{ng} \times \mathrm{gcn} / \mathrm{mole}) /$ $(\mathrm{bp} \times \mathrm{ng} / \mathrm{g} \times \mathrm{g} / \mathrm{mole}$ of $\mathrm{bp})]$. A standard curve of $1-10^{8}$ copies was used to determine copy number in each sample. The standard curve of each parasite was later compared with DNA extracted from blood with a known percentage of parasitized erythrocytes (\%PE, obtained from culture diluted in non-infected horse blood) to extrapolate from gene copies to infected red blood cells [20]. The cut-off for parasite detection was set as one gene copy, equivalent to $6.3 \times 10^{-5} \%$ PE for T. equi and 8 $\times 10^{-5} \%$ PE for B. caballi.

Parasite quantities of clinical versus subclinical cases and of T. equi versus B. caballi subclinical cases were compared using Mann-Whitney nonparametric statistical test. Receiver operating characteristic (ROC) curves were generated to establish cut-off values to differentiate parasitemia between clinical and subclinical cases of each parasite. The statistical analysis was performed in SPSS $22.0^{\circledR}$ software (IBM, Armonk, NY, USA).

\section{Results \\ Study population}

Infection with EP parasites was confirmed in 12 horses exhibiting clinical signs consistent with EP including fever, anemia, icterus and inappetence. In six of these cases parasites were identified in blood smear (others were not tested). In the 12 clinical cases, diagnostic PCR identified six cases of T. equi infection and six other cases of $B$. caballi infection. One of the T. equi cases was a fatal case of a neonatal filly born to a subclinically infected mare [27]. The ages of clinically infected horses ranged between newborn and 17 years-old. Sex, breed, mean age and mean PCV (PCV was available in ten cases) are listed in Table 1.

Samples from subclinical horses were collected from apparently healthy horses, including $40 \mathrm{~T}$. equi infected 
Table 1 Characteristics of the horses participated in this study

\begin{tabular}{|c|c|c|c|c|}
\hline & \multicolumn{2}{|c|}{ Babesia caballi } & \multicolumn{2}{|c|}{ Theileria equi } \\
\hline & Clinical & Subclinical & Clinical & Subclinical \\
\hline$n$ & 6 & 13 & 6 & 40 \\
\hline Mare & 3 & 2 & 4 & 16 \\
\hline Gelding & 3 & 10 & 2 & 23 \\
\hline Stallion & & 1 & & 1 \\
\hline Arabian & & & 1 & \\
\hline Tennessee walking horse & 1 & & 1 & \\
\hline Quarter horse & 3 & & 4 & 4 \\
\hline Warmblood & 1 & & & \\
\hline Andalusian & & 1 & & \\
\hline Pony & & & & 2 \\
\hline Mixed & 1 & 12 & & 34 \\
\hline Mean age & 6.20 & 6.90 & 12.17 & 9.17 \\
\hline Mean PCV & 19.75 & 30.70 & 19.42 & 33.82 \\
\hline
\end{tabular}

Note: The number $(n)$ of clinical and subclinical isolates analyzed in this study, sex and breed distribution, mean age and mean packed cell volume (PCV) are specified for each group

horses and 13 horses co-infected with both T. equi and $B$. caballi. The ages of the subclinically infected horses ranged between 2-22 years. Sex, breed, mean age and mean PCV (available in 59 cases) are listed in Table 1.

The mean PCV of clinically infected horses was significantly lower than of subclinically infected horses $(P<0.001,95 \%$ confidence interval, $\mathrm{CI}$ : $-17.41-11.24$ for T. equi and $P<0.001,95 \%$ CI: $-15.84-6.06$ for B. caballi, Table 1). The geographical distribution of the collection sites is presented in Additional file 3: Figure S1.

\section{Quantification of EP parasitemia in clinical versus subclinical horses}

Quantitative real time PCR was used to evaluate $T$. equi parasitemia in five clinically infected and seven, randomly selected, subclinically infected horses. Parasitemia of clinically infected animals ranged between 1998-59,813 ema-1 gene copies (mean: 21,033.6; SEM: $10,076.2$ ), equivalent to $0.12-3.8 \%$ parasitized erythrocytes (PE), while in subclinical animals it ranged between 5-81 gene copies (mean: 27.3; SEM: 11.2), equivalent to $0.0003-0.005 \%$ PE (Fig. 1a). The difference between parasite loads of clinical versus subclinical animals was statistically significant (Mann-Whitney, $P=0.003$ ). A diagnostic cut-off was calculated as 1040 gene copies or $0.066 \%$ PE with a sensitivity and specificity of $100 \%$ (ROC $\mathrm{AUC}=1,95 \%$ CI: $1, P=0.004$ ).

Quantitative real time PCR was used to evaluate $B$. caballi parasitemia in six clinically infected and seven, randomly selected, subclinically infected horses. Parasitemia of clinically infected animals ranged between

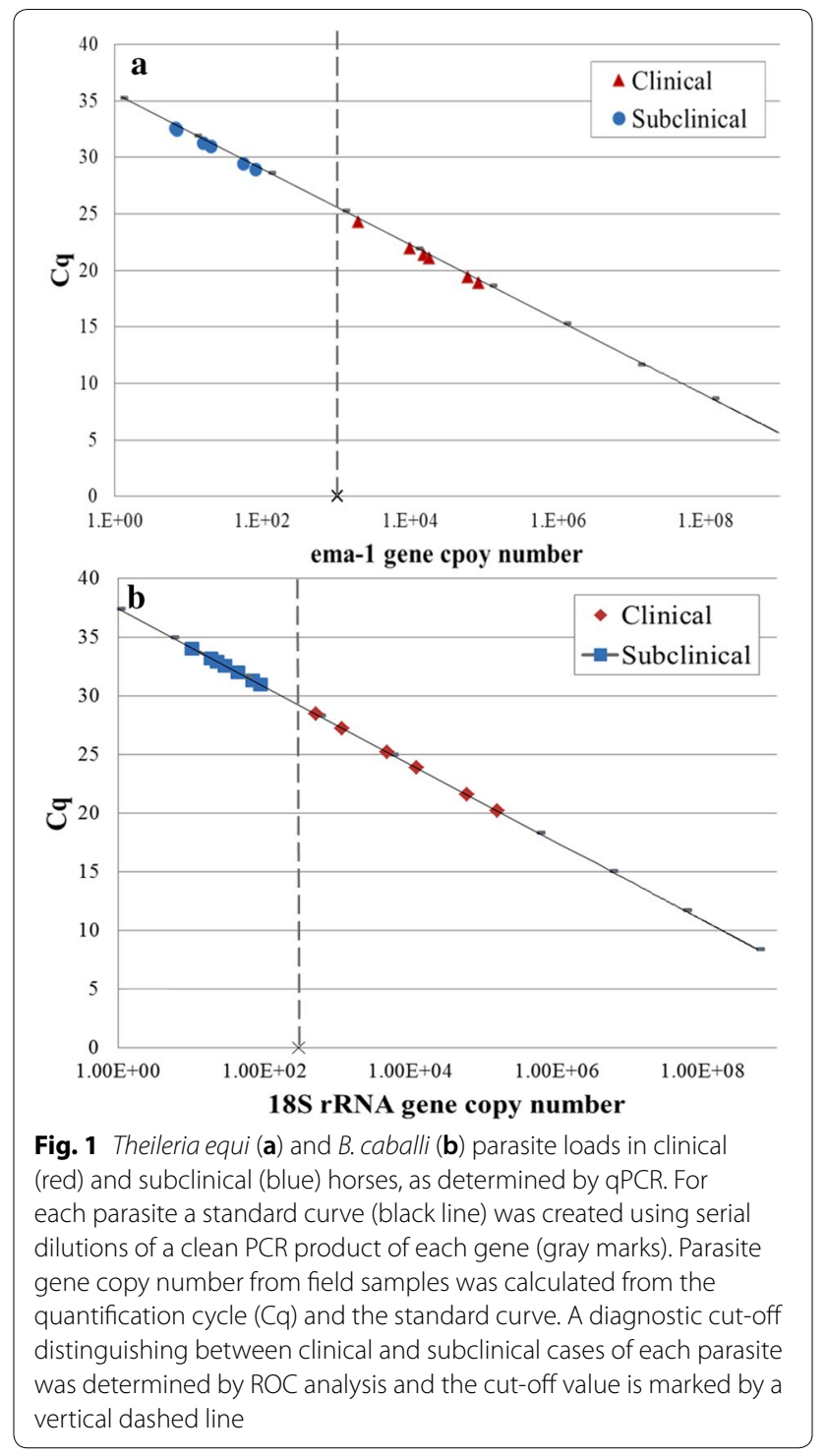

503-152,696 $18 S$ rRNA gene copies (mean: 38,348; SEM: $24,587.7$ ), equivalent to $0.007-2.11 \% \mathrm{PE}$, while in subclinical animals it ranged between 10-88 gene copies (mean: 40.5; SEM: 10.9), equivalent to $0.0001-0.0012 \% \mathrm{PE}$ (Fig. 1b). The difference between parasite loads of clinical versus subclinical animals was statistically significant $(P=0.001)$. A diagnostic cut-off was calculated as 296 gene copies or $0.004 \% \mathrm{PE}$ with a sensitivity and specificity of $100 \%$ (ROC AUC $=1,95 \%$ CI: $1, P=0.003$ ).

\section{Classification of T. equi and B. caballi based on 18S rRNA gene}

The $18 S$ rRNA gene was successfully amplified and sequenced in all T. equi clinical and subclinical samples (GenBank: MK392050-MK392061, 
MN611313-MN611352) and from all B. caballi clinical samples (GenBank: MK288106-MK288110, MN629354). All B. caballi-positive subclinical horses were co-infected with T. equi and, therefore, could not have been classified based on their $18 S$ rRNA gene.

Out of the five T. equi $18 S$ rRNA genotypes (A-E) $[6,28]$, all six clinical samples and $12(30 \%)$ subclinical samples were classified as genotype A; 23 (57.5\%) subclinical isolates were classified as genotype $\mathrm{D}$ and the remaining five $(12.5 \%)$ subclinical isolates were classified as genotype $C$ (Fig. 2a).

Of the two B. caballi $18 S$ rRNA genotypes (A and B, with genotype B subdivided into subgroups B1 and B2) [6], the six clinical samples were classified as genotype $\mathrm{B}$ : four samples as genotype B1 and two samples as genotype B2 (Fig. 3a).

\section{Classification of T. equi based on ema-1 and ema-2 genes}

Nine T. equi ema-1 PCR products were successfully amplified and sequenced from four clinical and five subclinical horses (GenBank: MK415929-MK415937). Out of the three T. equi ema-1 genotypes (A-C [14]), all samples were classified as genotype A (Fig. 2b).

Theileria equi ema-2 PCR products were successfully amplified and sequenced from all six clinical and five subclinical horses (GenBank: MN624965-MN624979). The 11 sequences obtained in this study were analyzed with all available T. equi ema-2 sequences on GenBank (20). Based on mean evolutionary distance (Additional file 4: Table S3), three genotypes were classified (A-C). The mean evolutionary distance within each genotype was under 0.004 base substitutions and the mean difference between groups was $0.011-0.059$ base substitutions. All clinical and three subclinical isolates were classified as genotype $\mathrm{A}$ and two subclinical isolates were classified as genotype $\mathrm{C}$ (Fig. 2c).

Of the nine samples classified as ema-2 genotype A, eight were also classified as $18 \mathrm{~S}$ rRNA genotype A and one as genotype $\mathrm{C}$. Of the two samples classified as ema2 genotype $\mathrm{C}$, one was $18 \mathrm{~S}$ rRNA genotype $\mathrm{C}$ and the other genotype D (Additional file 1: Table S1). The latter sequence was omitted from the phylogenetic analysis due to insufficient length.

\section{Classification of B. caballi rap-1 gene}

Babesia caballi rap-1 gene was successfully amplified and sequenced from 19 horses, six showing clinical signs of disease and 13 subclinical carriers (GenBank: MK346858-MK346873, MN635788). A long fragment of over $1400 \mathrm{bp}$ was obtained from all clinical and three subclinical cases; and a shorter fragment, between 250$1000 \mathrm{bp}$ from the remaining samples.
Of the two B. caballi rap-1 genotypes (A and B, with genotype A subdivided into subgroups A1 and A2) [12], four clinical and ten subclinical sequences obtained in this study were classified as genotype A1, while two clinical and three subclinical sequences were classified as genotype A2. All three subclinical isolates characterized as A2 originated from the same farm (Fig. 3b).

All clinical samples were classified based on both their $18 S$ rRNA and rap-1 genes and showed association between the two. All four samples that were characterized as $18 S$ rRNA genotype B1 were also classified as rap1 genotype $\mathrm{A} 1$, while the remaining two samples were $18 S$ rRNA B2 and rap-1 A2 (Additional file 1: Table S1).

\section{Discussion}

Diagnosis of EP as the cause of clinical disease can be challenging in endemic areas, where the percentage of serologically and molecularly positive horses is high, and the detection of parasites does not necessarily imply on the cause of non-specific clinical signs [29]. Therefore, in clinical cases suspected as EP, quantitative evaluation of parasite load, using molecular tools, may assist in determining a threshold for cause of disease decision. Here we demonstrate that clinically infected horses with either parasite of EP have significantly higher parasite loads and lower PCV than subclinically infected horses. This is intuitive, as merozoite replication in erythrocytes causes hemolysis, the main clinical manifestation of EP. Thus, higher parasite loads may induce increased hemolysis that will be reflected in lower PCV. Parasite loads of both clinical and subclinical horses were generally lower in cases of B. caballi infection than in cases of T. equi infection. This may explain the milder clinical disease in B. caballi infections compared to T. equi infections, and to the possible natural clearance of $B$. caballi parasitemia without treatment, while T. equi carriage is usually lifelong $[1,2]$.

To determine whether T. equi is the probable cause of disease in suspected clinical cases, we established a clear cut-off $(P<0.001)$ between clinical $(0.12-3.8 \%$ $\mathrm{PE})$ and subclinical $\left(3 \times 10^{-4}-5 \times 10^{-3} \% \mathrm{PE}\right)$ cases. The parasitemia values in our study concur with published subclinical range (1.99-1000 parasites per $\mu$ l blood [30], equivalent to $2.2 \times 10^{-5}$ to $0.011 \% \mathrm{PE}$ ). In clinical cases, T. equi parasitemia ranges between 1-7\% PE, and may reach up to $95 \%[1,31]$; however, we had clinical cases with parasitemia as low as $0.12 \% \mathrm{PE}$, which also presented with a low PCV. Three of six clinically $B$. caballi infected horses showed parasitemia below the documented range $(0.1-10 \% \mathrm{PE})[1,31]$. Parasitemia in subclinical carriers of $B$. caballi ranged between $0.0001-$ $0.0012 \% \mathrm{PE}$, which was significantly lower than the clinical cases $(P<0.001)$. To the best of our knowledge, no 


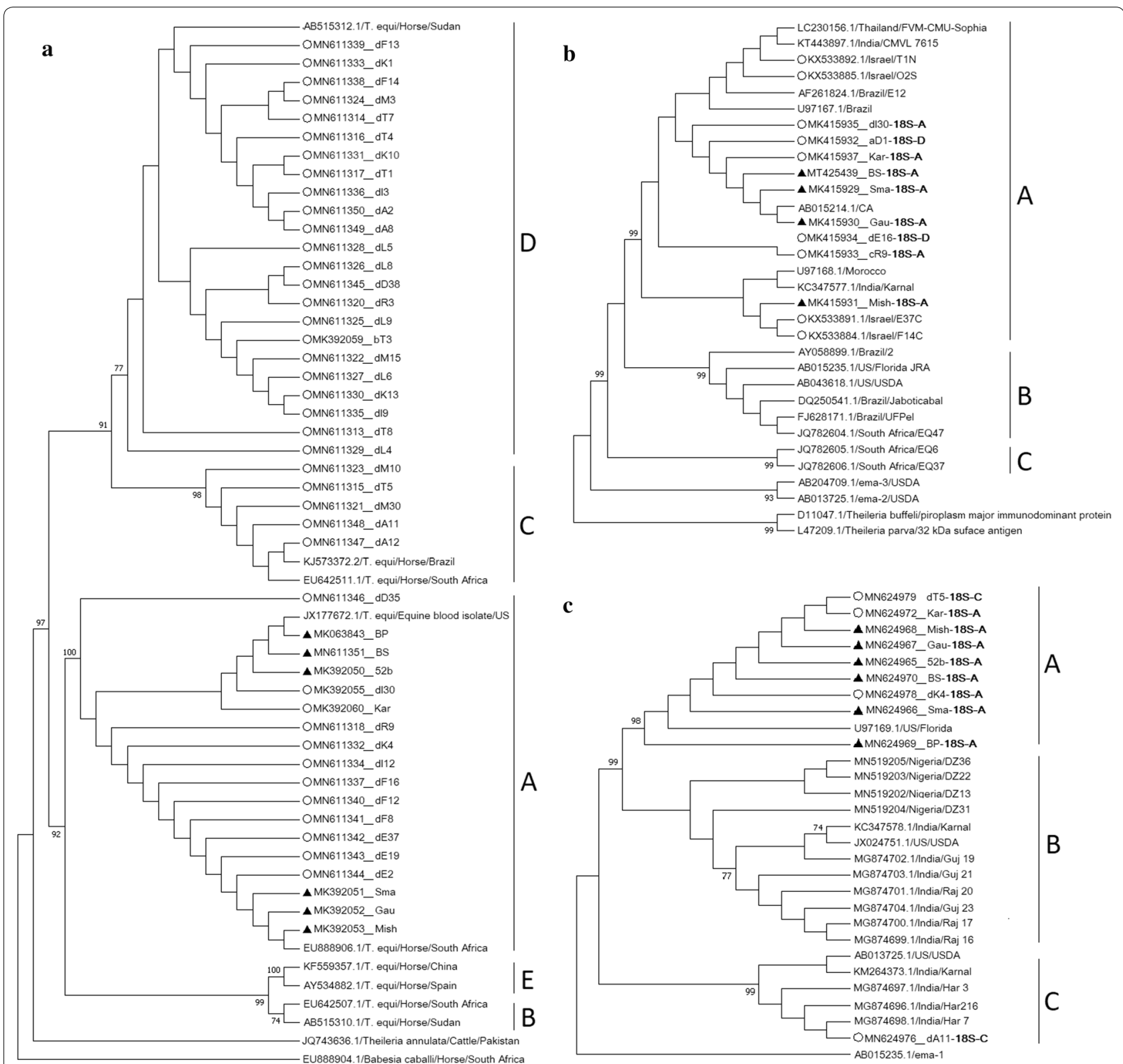

Fig. 2 Phylogenetic analysis of T. equi sequences obtained from clinically infected horses (triangles) and subclinically infected horses (open circles) using three genes (sample names as detailed in Additional file 1:Table S1). a Analysis of 1079 nucleotide positions of T. equi 185 rRNA gene, from 6 clinical and 40 subclinical samples, along with additional published sequences (GenBank ID/parasite/host/location). The phylogenetic tree was constructed based on the Tamura-Nei model with gamma distribution $(+\mathrm{G})$. b Analysis of 400 nucleotide positions of T. equi ema-1 gene sequences obtained from four clinical and five subclinical samples, along with additional published sequences (GenBank ID/parasite/host/location). The classification of each sample according to its 185 rRNA gene is states near the sample name (-18SX). The phylogenetic tree was constructed based on the Kimura 2-parameter model with consideration on invariable sites (+I). c Analysis of 782 nucleotide positions of T. equi ema-2 gene sequences obtained from six clinical and four subclinical samples, along with all 19 additional published sequences (GenBank ID/parasite/host/ location). The classification of each sample according to its 185 rRNA gene is states near the sample name (-18SX). The phylogenetic tree was constructed by based on the Kimura 2-parameter model with consideration on invariable sites $(+1)$. All phylogenetic trees were constructed using maximum likelihood method and 1000 bootstrap replicates. The percentage of trees in which the associated samples clustered together is shown next to the branches when it was above $70 \%$. The analysis was constructed in MEGA7 


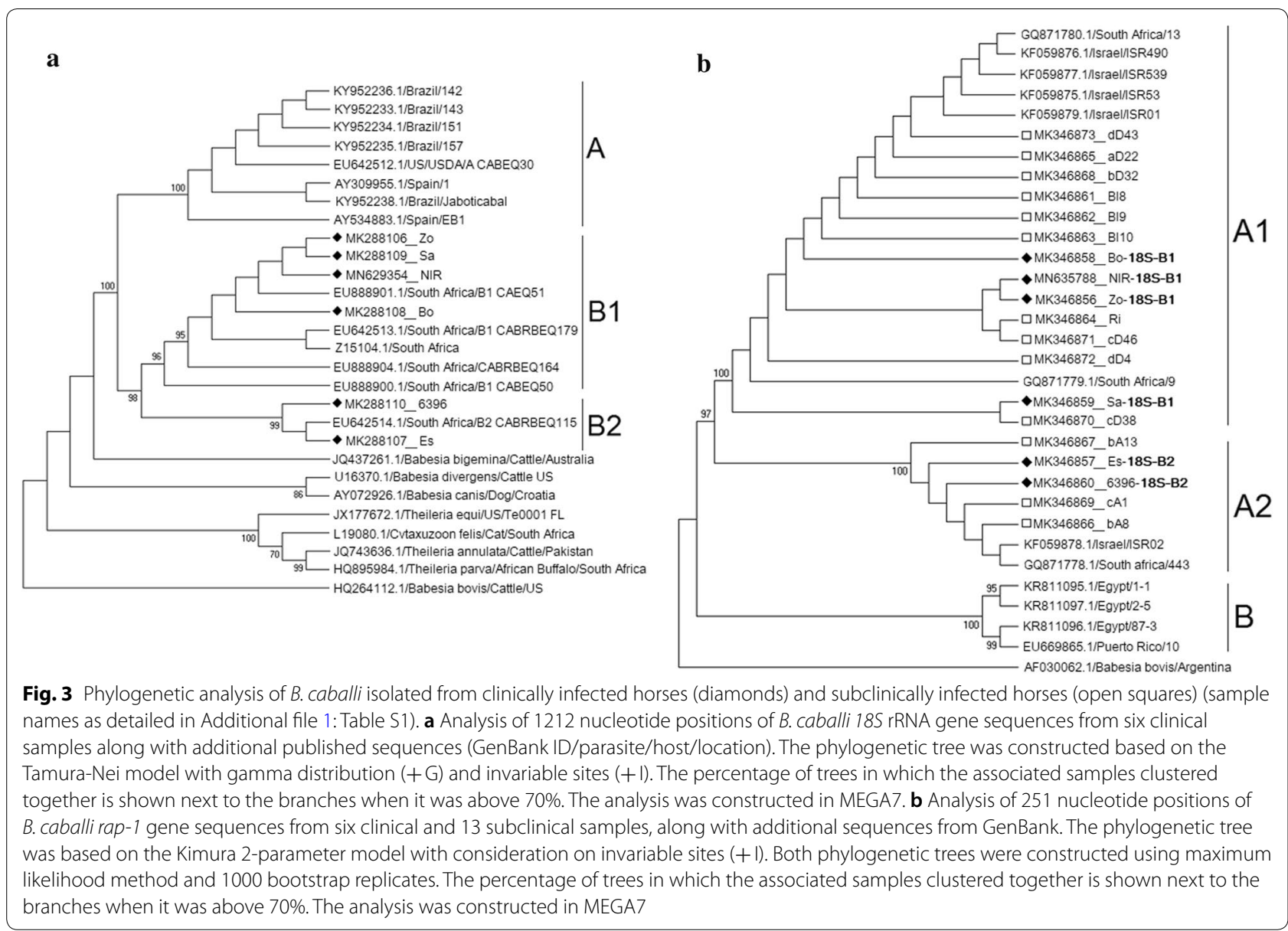

previous study quantified $B$. caballi parasitemia in subclinical horses. Although in this group, the difference between clinical and subclinical parasitemia was less distinct, it still resulted in a lower PCV, and allowed us to establish a cut-off value to identify $B$. caballi as the probable cause of clinical disease. Despite the limited number of cases included in the quantitative analysis, the highly significant results may serve as first indication that qPCR can be used as a diagnostic tool. Additional data should be collected to validate this method for clinical use.

All six clinical cases which originated from different farms and geographical locations were classified as T. equi $18 S$ rRNA genotype A. The A genotype is not the most prevalent in Israel (30\% of subclinical horses in this study, $33 \%$ in a previous study), and is rarely found in highly endemic farms [3]. Thus, although the number of cases was limited, genotype A may be associated with clinical disease. Genotype A was previously isolated from horses in both endemic and non-endemic countries $[3,6,28,32]$, it was isolated in two outbreaks in the USA [33], and was found to be associated with clinical and seropositive cases in Italy [19]. Interestingly, genotype A was the predominant genotype isolated from ticks collected from horses in Israel, including five different tick species of three genera. Some of these ticks were collected in farms in which this genotype was not isolated from horses [34, 35]. Interestingly, we found an association between T. equi $18 \mathrm{~S}$ rRNA genotype and farm management (unpublished results). It is possible that this genotype is more adapted to the tick vector environment but encounters an active barrier during transmission to the host. Genotype A may also be less adapted to the host and may thus be more likely to result in clinical disease, whilst genotypes B, C and $\mathrm{D}$ are more likely to result in subclinical infection [19]. With the recent concerns regarding the classification of Theileria species according to the $18 S$ rRNA gene [7, 8], it is possible that this "A genotype" is the cause of the "classic" equine theileriosis, while other genotypes may represent closely related, less pathogenic, species or subspecies. More comprehensive genetic investigation of different genotypes is required to support this hypothesis. 
In an attempt to partially address these issues, we classified T. equi according to three different genes: $18 \mathrm{~S}$ rRNA, ema-1 and ema-2, as the last two loci had a sufficient number of published sequences for comparative analysis. However, we could not amplify ema-1 and ema-2 from all samples, probably due to polymorphism in the primer sites or the sensitivity of the PCR assay, and most of the successfully sequenced amplicons were from isolates of $18 S$ rRNA genotype A, as previously reported [19]. The overrepresented $18 S$ rRNA genotype A may be the result of the higher parasitemia in the clinically infected horses, enabling better detection by PCR. Nevertheless, using qPCR, ema-1 gene was detected in all samples, strengthening parasite identification.

The $18 S$ rRNA classification in subclinical horses resulted in the detection of genotypes D (57.5\%), A (30\%) and $C(12.5 \%)$ as previously described in our area [3], thus strengthening the statistical power of the data with a larger sample size. Sequence analysis of both ema-1 and ema-2 did not reveal much polymorphism in these loci within a geographical area, as has been previously demonstrated [3, 14, 16-19]. Only 16 ema-2 sequences were available for classification, mostly from India and the USA along with four sequences from Nigeria [36]. Although this gene had low variability, we identified three distinct genotypes, which also differ in their amino acid sequences. This variability may be important if it affects immune response and may also lower the sensitivity of the ema-2 based ELISA assays [18].

Genetic classification of $B$. caballi is limited, with two $18 S$ rRNA genotypes identified in South Africa [6]. We were unable to amplify this gene from the subclinical horses, since all were co-infected with T. equi, and the primers are not species specific. Therefore, we used the rap-1 gene which is specific to $B$. caballi and is fairly conserved, although with some heterogenicity between American and Asian-African strains [12-14, 19]. Two $18 S$ rRNA sub-genotypes were identified in clinical cases, which correlated with the rap-1 sub-genotypes of the same samples. Comparison of the rap-1 gene between clinical and subclinical cases did not reveal differences in parasite genotypes in relation to clinical disease.

The main limitation of this study was the small sample size of clinical cases. The number of clinical cases was limited mostly due to the relatively low occurrence of clinical cases in Israel. In addition, the relatively low prevalence of $B$. caballi in combination with high co-infection rates with $T$. equi prevented the establishment of a single-positive control group for this parasite and interfered with the ability to obtain good quality sequences of its $18 S$ rRNA gene. Lastly, in cases of T. equi infection, variations in the sensitivity of the PCR assays of the different loci did not allow the comprehensive analysis of all three genes from all samples. Nevertheless, the current groundwork brings unique quantitative data to be further supported by future studies.

\section{Conclusions}

This study provides preliminary results supporting the use of quantitative molecular tools which assess parasite loads to help clinicians decide whether EP is the cause of the presenting clinical signs. In addition, T. equi genotype A (based on $18 S$ rRNA classification) was associated with clinical disease, while no such association was found for B. caballi. Future studies on parasites classification should be established in order to distinguish between closely related organisms or genotypes which may differ in their pathogenicity.

\section{Supplementary information}

Supplementary information accompanies this paper at https://doi. org/10.1186/s13071-020-04133-y.

Additional file 1: Table S1. Detailed description of the study population, their clinical status and associated parasites of B. caballi and T. equi.

Additional file 2: Table S2. The PCR primers and probes used in this study.

Additional file 3: Figure S1. The geographical distribution of the collection sites of all clinical and subclinical equine cases of T. equi and B. caballi infection.

Additional file 4: Table S3. Estimates of the evolutionary divergence within and between T. equi ema-2 genotypes.

\section{Abbreviations}

ema: equi merozoite antigen; EP: equine piroplasmosis; gcn: gene copy number; PCV: packed cell volume; PE: parasitized erythrocytes; TS: total solids; rap: rhoptry associated protein.

\section{Acknowledgments}

The authors wish to thank the farm owners for their willingness to participate in the surveillance study, the equine clinicians for collecting blood samples from clinical cases and Dr Monica L. Mazuz for providing the cultured parasites.

\section{Authors' contributions}

YG and AS conceived, designed and supervised the study. STL designed the study, conducted the field work, analyzed the data and drafted the manuscript. STL, HL, YK and MS preformed the lab experiments. All authors read and approved the final manuscript.

Funding

Not applicable.

\section{Availability of data and materials}

The datasets supporting the conclusions of this article are included within the article and its additional files.

\section{Ethics approval and consent to participate}

All samples were obtained with owner's permission and the study was approved by the Research Committee of the Koret School of Veterinary Medicine, Veterinary Teaching Hospital (KSVM-VTH/23_2014).

Consent for publication

Not applicable. 


\section{Competing interests}

The authors declare that they have no competing interests.

Received: 6 January 2020 Accepted: 11 May 2020

Published online: 20 May 2020

\section{References}

1. Rothschild CM. Equine piroplasmosis. J Equine Vet Sci. 2013;23:115-20.

2. Wise LN, Kappmeyer LS, Mealey RH, Knowles DP. Review of equine piroplasmosis. J Vet Intern Med. 2013;27:1334-46.

3. Ketter-Ratzon D, Tirosh-Levy S, Nachum-Biala Y, Saar T, Qura'n L, Zivotofsky D, et al. Characterization of Theileria equi genotypes in horses in Israel, the Palestinian Authority and Jordan. Ticks Tick Borne Dis. 2017;8:499-505.

4. Scoles GA, Ueti MW. Vector ecology of equine piroplasmosis. Annu Rev Entomol. 2015;60:561-80.

5. Kuttler K, Gipson C, GoffW, Johnson L. Experimental Babesia equi infection in mature horses. Am J Vet Res. 1986;47:1668-70.

6. Bhoora R, Franssen L, Closthuizen MC, Guthrie AJ, Zweygarth E, Penzhorn $\mathrm{BL}$, et al. Sequence heterogeneity in the $18 \mathrm{~S}$ rRNA gene within Theileria equi and Babesia caballi from horses in South Africa. Vet Parasitol. 2009;159:112-20.

7. Dahmana H, Amanzougaghene N, Davoust B, Normand T, Carette O, Demoncheaux J-P, et al. Great diversity of Piroplasmida in Equidae in Africa and Europe, including potential new species. Vet Parasitol Reg Stud Reports. 2019;18:100332.

8. Knowles DP, Kappmeyer LS, Haney D, Herndon DR, Fry LM, Munro JB, et al. Discovery of a novel species, Theileria haneyi n. sp., infective to equids, highlights exceptional genomic diversity within the genus Theileria: implications for apicomplexan parasite surveillance. Int J Parasitol. 2018;48:679-90.

9. Mans BJ, Pienaar R, Latif AA. A review of Theileria diagnostics and epidemiology. Int J Parasitol Parasites Wildl. 2015;4:104-18.

10. Bhoora R, Zweygarth E, Guthrie AJ, Franssen L, Jongejan F, Oosthuizen MC, et al. Characterisation of South African Theileria equi and Babesia caballi isolates based on 185 rRNA gene sequences. J S Afr Vet Assoc. 2009;80:116.

11. Bhoora R, Quan M, Zweygarth E, Guthrie AJ, Prinsloo SA, Collins NE. Sequence heterogeneity in the gene encoding the rhoptry-associated protein-1 (RAP-1) of Babesia caballi isolates from South Africa. Vet Parasitol. 2010;169:279-88.

12. Rapoport A, Aharonson-Raz K, Berlin D, Tal S, Gottlieb Y, Klement E, et al. Molecular characterization of the Babesia caballi rap-1 gene and epidemiological survey in horses in Israel. Infect Genet Evol. 2014;23:115-20.

13. Mahmoud MS, El-Ezz NT, Abdel-Shafy S, Nassar SA, El Namaky AH, Khalil WK, et al. Assessment of Theileria equi and Babesia caballi infections in equine populations in Egypt by molecular, serological and hematological approaches. Parasit Vectors. 2016;9:260.

14. Bhoora R, Quan M, Matjila PT, Zweygarth E, Guthrie AJ, Collins NE. Sequence heterogeneity in the equi merozoite antigen gene (ema-1) of Theileria equi and development of an ema-1-specific TaqMan MGB assay for the detection of T. equi. Vet Parasitol. 2010;172:33-45.

15. Kappmeyer LS, Thiagarajan M, Herndon DR, Ramsay JD, Caler E, Djikeng A, et al. Comparative genomic analysis and phylogenetic position of Theileria equi. BMC Genomics. 2012;13:603.

16. Wise LN, Kappmeyer LS, Knowles DP, White SN. Evolution and diversity of the EMA families of the divergent equid parasites, Theileria equi and $T$. haneyi. Infect Genet Evol. 2019;68:153-60.

17. Knowles DP Jr, Kappmeyer LS, Stiller D, Hennager SG, Perryman LE. Antibody to a recombinant merozoite protein epitope identifies horses infected with Babesia equi. J Clin Microbiol. 1992;30:3122-6.

18. Kumar S, Kumar R, Gupta AK, Yadav SC, Goyal SK, Khurana SK, et al. Development of EMA-2 recombinant antigen based enzyme-linked immunosorbent assay for seroprevalence studies of Theileria equi infection in Indian equine population. Vet Parasitol. 2013;198:10-7.
19. Manna G, Cersini A, Nardini R, Del Pino LEB, Antognetti V, Zini M, et al. Genetic diversity of Theileria equi and Babesia caballi infecting horses of central-southern Italy and preliminary results of its correlation with clinical and serological status. Ticks Tick Borne Dis. 2018;9:1212-20.

20. Tirosh-Levy S, Gottlieb Y, Arieli O, Mazuz ML, King R, Horowitz I, et al. Genetic characteristics of Theileria equi in zebras, wild and domestic donkeys in Israel and the Palestinian Authority. Ticks Tick Borne Dis. 2020;11:101286.

21. Alhassan A, Pumidonming W, Okamura M, Hirata H, Battsetseg B, Fujisaki $K$, et al. Development of a single-round and multiplex PCR method for the simultaneous detection of Babesia caballi and Babesia equi in horse blood. Vet Parasitol. 2005;129:43-9.

22. Steinman A, Zimmerman T, Klement E, Lensky IM, Berlin D, Gottlieb Y, et al. Demographic and environmental risk factors for infection by Theileria equi in 590 horses in Israel. Vet Parasitol. 2012;187:558-62.

23. Matjila PT, Leisewitz AL, Oosthuizen MC, Jongejan F, Penzhorn BL. Detection of a Theileria species in dogs in South Africa. Vet Parasitol. 2008;157:34-40.

24. Oosthuizen MC, Zweygarth E, Collins NE, Troskie M, Penzhorn BL. Identification of a novel Babesia sp. from a sable antelope (Hippotragus niger Harris, 1838). J Clin Microbiol. 2008;46:2247-51.

25. Kumar S, Stecher G, Tamura K. MEGA7: Molecular Evolutionary Genetics Analysis Version 7.0 for bigger datasets. Mol Biol Evol. 2016;33:1870-4.

26. Bhoora R, Quan M, Franssen L, Butler CM, van der Kolk JH, Guthrie AJ, et al. Development and evaluation of real-time PCR assays for the quantitative detection of Babesia caballi and Theileria equi infections in horses from South Africa. Vet Parasitol. 2010;168:201-11.

27. Levi MM, Tirosh-Levy S, Dahan R, Berlin D, Steinman A, Edery N, et al. First detection of diffuse and cerebral Theileria equi infection in neonatal filly. J Equine Vet Sci. 2018;60:23-8.

28. Munkhjargal T, Sivakumar T, Battsetseg B, Nyamjargal T, Aboulaila M, Purevtseren $B$, et al. Prevalence and genetic diversity of equine piroplasms in Tov province, Mongolia. Infect Genet Evol. 2013;16:178-85.

29. Camino E, Dorrego A, Carvajal KA, Buendia-Andres A, de Juan L, Dominguez L, et al. Serological, molecular and hematological diagnosis in horses with clinical suspicion of equine piroplasmosis: pooling strengths. Vet Parasitol. 2019;275:108928.

30. Ueti MW, Palmer GH, Kappmeyer LS, Statdfield M, Scoles GA, Knowles DP. Ability of the vector tick Boophilus microplus to acquire and transmit Babesia equi following feeding on chronically infected horses with lowlevel parasitemia. J Clin Microbiol. 2005;43:3755-9.

31. de Waal DT. Equine piroplasmosis: a review. Br Vet J. 1992;148:6-14.

32. Sant C, Allicock OM, d'Abadie R, Charles RA, Georges K. Phylogenetic analysis of Theileria equi and Babesia caballi sequences from thoroughbred mares and foals in Trinidad. Parasitol Res. 2019;118:1171-7.

33. Hall CM, Busch JD, Scoles GA, Palma-Cagle KA, Ueti MW, Kappmeyer LS, et al. Genetic characterization of Theileria equi infecting horses in North America: evidence for a limited source of U.S. introductions. Parasit Vectors. 2013;6:35

34. Tirosh-Levy S, Gottlieb Y, Apanaskevich DA, Mumcuoglu KY, Steinman A. Species distribution and seasonal dynamics of equine tick infestation in two Mediterranean climate niches in Israel. Parasit Vectors. 2018;11:546.

35. Tirosh-Levy S, Steinman A, Einhorn A, Apanaskevich DA, Mumcuoglu KY, Gottlieb Y. Potential tick vectors for Theileria equi in Israel. Med Vet Entomol. 2020. https://doi.org/10.1111/mve.12435.

36. Idoko SI, Tirosh-Levy S, Mazuz ML, Adam BM, Garba BS, Nafarnda DW, et al. Genetic characterization of piroplasms in donkeys and horses from Nigeria. Animals (Besel). 2020;10:E324.

\section{Publisher's Note}

Springer Nature remains neutral with regard to jurisdictional claims in published maps and institutional affiliations. 